Preprint typeset in JHEP style. - HYPER VERSION

MCTP-01-26

hep-th/0106046

\title{
The Reduced Open Membrane Metric
}

\author{
Jan Pieter van der Schaar \\ Michigan Center for Theoretical Physics \\ Randall Laboratory, Department of Physics, University of Michigan \\ Ann Arbor, MI 48109-1120, USA \\ E-mail: jpschaar@umich.edu
}

\begin{abstract}
We discuss the reduction of the open membrane metric and determine the (previously unknown) conformal factor. We also construct $S L(2, R)$ invariant open string metrics and complex open string coupling constants by reducing the open membrane metric on a 2-torus. In doing so we also clarify some issues on manifest $S L(2, R)$ symmetry of the D3-brane. We remark on the consequences of our results for the recently conjectured existence of decoupled $(p, q)$ non-commutative open string theories in type IIB string theory.
\end{abstract}

Keywords: M-theory, D-branes, Non-Commutative Geometry. 


\section{Contents}

1. Introduction 1

2. M5-brane preliminaries 3

3. The open membrane metric on the circle 5

4. The open membrane metric on the torus 9

5. Summary and discussion 16

\section{Introduction}

A widespread interest in non-commutative theories from the perspective of string theory started with the paper of Seiberg and Witten [1]. They realized that, among other things, open strings probing the D-brane are most naturally described using open string moduli which depend on the 2 -form $\mathcal{F}$ on the D-brane; the open string metric $\left(G_{o s}^{-1}\right)_{a b}$ and the open string coupling constant $\lambda_{o s}$. As first discussed in [2], this situation was generalized to include the M5-brane of M-theory, which involved a conjectured open membrane $(\mathrm{OM})$ metric which depended on the nonlinearly self-dual 3 -form $\mathcal{H}$ on the M5-brane.

In a somewhat different context it was shown in [3, 4] that both the open string and the open membrane metric provide the propagation cone for all D3- and M5-brane degrees of freedom, which always lies inside the bulk Einstein light-cone. Consequently they also showed explicitly that all equations of motion on the M5-brane and the D3-brane can be conveniently written using a symmetric tensor that is in the same conformal class as the open string and open membrane (co-) metric, the so-called Boillat metric.

Attempts to decouple D-brane theories from the bulk supergravity were at first only successful for magnetic field strengths giving rise to non-commutative gauge theories [1]. To avoid problems with unitarity in spatio-temporal non-commutative field theory [5, 6] one was lead to the discovery of decoupled non-commutative open string theories (NCOS) on D-branes [7, 8], forcing one to take a critical limit for the electric field 
strength. By considering a decoupling limit where the electric 3-form field strength on the M5-brane becomes (nearly) critical this lead to the introduction of OM-theory [9, 10], which can be understood as the mother of all (spatially and spatio-temporally) non-commutative theories.

In [10] the conformal factor in front of the open membrane metric was fixed using a decoupling argument: the open membrane metric $\left(G_{O M}^{-1}\right)_{a b}$ and the $D=11$ Planck length $\ell_{p}$ should define a finite (non-commutative) length scale $\ell_{g}$ in the OM-theory decoupling limit. This allowed one to fix the conformal factor, but only up to terms that vanish in the decoupling limit. In this paper we would like to determine the conformal factor without ever considering a decoupling limit. Our guiding principle will be that we want the open membrane metric $\left(G_{O M}^{-1}\right)_{a b}$ to reduce to the open string metric $\left(G_{o s}^{-1}\right)_{a b}$ and the open string coupling constant $\lambda_{o s}$. As we will see this will enable us, using reduction ansatze analogous to the bulk M-theory/IIA relations, to fix the conformal factor.

Reducing the open membrane metric to an expression depending only on the 2form $\mathcal{F}$ is nontrivial because of the nonlinear self-duality equation on the M5-brane and consequently, the nonlinear duality equation on the D4-brane (described by nonlinear Dirac-Born-Infeld (DBI) theory, for a review see [11]). The reduction of the open membrane metric was performed in [12, 13], but to obtain their result we will proceed in a slightly different (and less general) manner. More importantly, they were unaware at that time of the interpretation of their symmetric tensor as the open membrane metric and therefore did not consider the internal component of that tensor upon reduction, which we will now interpret as the open string coupling constant. So keeping these newly acquired insights in mind and redoing their calculation [12, 13], simplifying matters by imposing a constraint that will restrict our attention to rank 2 solutions of the 2 -form $\mathcal{F}$, we are able to fix the conformal factor of the open membrane metric as to give us both the open string metric and the open string coupling constant upon (double dimensional) reduction of the M5-brane.

After fixing the conformal factor we reduce the open membrane metric on a 2-torus giving an $S L(2, R)$ invariant open string metric and introducing a complex open string coupling constant as the modular parameter of the torus as seen by the open membrane. We find that generically the situation on the D3-brane is very similar to the M5-brane in the sense that the doublet of 2-form field strengths has to satisfy a nonlinear self-duality equation. By $S L(2, R)$ rotating into a special frame we can reproduce the SeibergWitten results, at the cost of giving up manifest $S L(2, R)$ invariance. These results are of course intimately connected to previous investigations of $S L(2, R)$ invariance on the D3-brane [14, 15, 16, 17, 18], but we will make full use of the open membrane metric idea in the sense that the $S L(2, R)$ symmetry is generated by the open membrane torus, 
instead of the usual 'bulk' torus. In that way we will also be connecting to work done with respect to the $S L(2, R)$ symmetry of NCOS theories on the D3-brane [19, 20, 21. In the same section we also comment on the decoupling limit that would give rise to $(p, q)$ non-commutative open strings that were discussed recently in 22, 23]. We end with some conclusions, remarks and possible future extensions of our work.

\section{M5-brane preliminaries}

Our starting point ${ }^{1}$ will be the following six-dimensional symmetric tensor defined on a single (abelian) M5-brane $(\hat{a}, \hat{b} \in(0,1, \ldots, 5))$,

$$
\hat{C}^{\hat{a} \hat{b}}=\frac{1}{K}\left[\left(1+\frac{1}{12} \hat{\mathcal{H}}^{2}\right) \hat{g}^{\hat{a} \hat{b}}-\frac{1}{4}\left(\hat{\mathcal{H}}^{2}\right)^{\hat{a} \hat{b}}\right],
$$

which depends on the gauge invariant 3-form field strength $\hat{\mathcal{H}}=d \hat{B}+\hat{C}$, where $\hat{B}$ is a 2-form gauge field living on the M5-brane and $\hat{C}$ is the 3-form gauge field of $D=11$ supergravity. We also used $\left(\hat{\mathcal{H}}^{2}\right)^{\hat{a} \hat{b}} \equiv \hat{\mathcal{H}}^{\hat{a} \hat{c}} \hat{d} \hat{\mathcal{H}}^{\hat{b}}{ }_{\hat{c} \hat{d}}$ and introduced a function $K$ equal to

$$
K=\sqrt{1+\frac{1}{24} \hat{\mathcal{H}}^{2}} .
$$

This tensor is conformal to the open membrane co-metric ${ }^{2}$ and was shown to reduce to the so-called Boillat metric of nonlinear DBI electrodynamics in [4].

The equations of motion of the tensor multiplet, the nonlinear self-duality equation and the energy momentum tensor on the M5-brane can all be conveniently written using this tensor (2.1) [4] (which implies that $C^{-1}$ defines the propagation cone for all perturbative degrees of freedom). For our purposes we only need the nonlinear self-duality equation the 3 -form $\hat{\mathcal{H}}$ satisfies on the M5-brane, which is

$$
\hat{C}_{\hat{a}}{ }^{\hat{d}} \hat{\mathcal{H}}_{\hat{d} \hat{b} \hat{c}}=\frac{\sqrt{-\operatorname{det} \hat{g}}}{3 !} \epsilon_{\hat{a} \hat{b} \hat{c} \hat{d} \hat{e} \hat{f}} \hat{\mathcal{H}}^{\hat{d} \hat{e} \hat{f}}
$$

For ease of computation we will from now on assume that the M5-brane worldvolume is flat, so $\hat{g}^{\hat{a} \hat{b}}=\hat{\eta}^{\hat{a} \hat{b}}$. In 12, 13 many of the calculations made use of relating the 3 -form $\hat{\mathcal{H}}$ to another (unphysical) 3 -form $\hat{h}$ which satisfies a linear self-duality equation. If possible, we would like to avoid using the linearly self-dual 3-form $\hat{h}$, but we are going

\footnotetext{
${ }^{1}$ We use a mostly plus signature convention for the metric and the 3 -form fields are dimensionless. We also use hats to distinguish the $D=6$ fields and indices from the $D=5$ fields and indices.

${ }^{2}$ Because indices are lowered and raised with the usual metric $\hat{g}_{\hat{a} \hat{b}}$, we have to make a clear distinction between inverse open brane metrics and co-metrics, i.e. $\left(\hat{G}^{-1}\right)_{\hat{a} \hat{b}} \neq \hat{G}_{\hat{a} \hat{b}}$.
} 
to use it to deduce a constraint on $\left(\hat{\mathcal{H}}^{4}\right)^{\hat{a} \hat{b}} \equiv\left(\hat{\mathcal{H}}^{2}\right)^{\hat{a} \hat{c}}\left(\hat{\mathcal{H}}^{2}\right)^{\hat{b}}{ }_{\hat{c}}$, which will turn out to be useful.

The two 3-forms are related in the following way

$$
\hat{h}_{\hat{a} \hat{b} \hat{c}}=\frac{1}{4} \hat{m}_{\hat{a}}^{\hat{d}} \hat{\mathcal{H}}_{\hat{d} \hat{b} \hat{c}}
$$

with

$$
\begin{aligned}
\hat{m}^{\hat{a} \hat{b}} & \equiv \hat{\eta}^{\hat{a} \hat{b}}-2 \hat{k}^{\hat{a} \hat{b}} \\
\hat{k}^{\hat{a} \hat{b}} & \equiv \hat{h}^{\hat{a}}{ }_{c \hat{d}} \hat{h}^{\hat{b} \hat{c} \hat{d}} .
\end{aligned}
$$

Because $\hat{h}$ satisfies a linear self-duality equation we find first of all that $\operatorname{Tr} \hat{k}^{\hat{a} \hat{b}} \equiv \hat{\eta}_{\hat{a} \hat{b}} \hat{k}^{\hat{a} \hat{b}}=$ 0 and we also deduce

$$
\begin{aligned}
\hat{h}_{\hat{a} \hat{b} \hat{e}} \hat{h}^{\hat{c} \hat{d} \hat{e}} & =\frac{1}{4} \hat{\delta}_{[\hat{a}}^{[\hat{c}} \hat{k}_{\hat{b}]}^{\hat{d}]} \\
\hat{k}_{\hat{a}}{ }^{\hat{c}} \hat{k}_{\hat{c}}^{\hat{b}} & =\frac{1}{6} \hat{k}^{2} \hat{\delta}_{\hat{a}}^{\hat{b}} .
\end{aligned}
$$

Using (2.7) it is straightforward to calculate the inverse of $\hat{m}$, which is equal to

$$
\left(\hat{m}^{-1}\right)^{\hat{a} \hat{b}}=\frac{1}{1-\frac{2}{3} \hat{k}^{2}}\left(\hat{\eta}^{\hat{a} \hat{b}}+2 \hat{k}^{\hat{a} \hat{b}}\right) .
$$

To find a constraint on $\left(\hat{\mathcal{H}}^{4}\right)^{\hat{a} \hat{b}}$ we replace $\hat{h}$ by $\hat{\mathcal{H}}$ in $(2.6)$ giving us

$$
\hat{\mathcal{H}}_{\hat{a} \hat{b} \hat{e}} \hat{\mathcal{H}}^{\hat{c} \hat{d} \hat{e}}=4 \frac{\frac{2}{3} \hat{k}^{2}}{\left(1-\frac{2}{3} \hat{k}^{2}\right)^{2}} \hat{\delta}_{[\hat{a}}^{[\hat{c}} \hat{\delta}_{\hat{b}]}^{\hat{d}]}+\frac{4}{\left(1-\frac{2}{3} \hat{k}^{2}\right)^{2}} \hat{k}_{[\hat{a}}^{[\hat{c}} \hat{k}_{\hat{b}]}^{\hat{d}]}+4 \frac{1+\frac{2}{3} \hat{k}^{2}}{\left(1-\frac{2}{3} \hat{k}^{2}\right)^{2}} \hat{\delta}_{[\hat{a}}^{[\hat{c}} \hat{k}_{\hat{b}]}^{\hat{d}]} .
$$

Tracing this equation once allows us to express $\hat{k}^{\hat{a} \hat{b}}$ as

$$
\hat{k}^{\hat{a} \hat{b}}=\frac{1}{16} \frac{\left(1-\frac{2}{3} \hat{k}^{2}\right)^{2}}{1+\frac{2}{3} \hat{k}^{2}}\left[\left(\hat{\mathcal{H}}^{2}\right)^{\hat{a} \hat{b}}-\frac{1}{6} \hat{\mathcal{H}}^{2} \hat{\eta}^{\hat{a} \hat{b}}\right]
$$

and tracing again gives

$$
\hat{\mathcal{H}}^{2}=96\left(\frac{\frac{2}{3} \hat{k}^{2}}{\left(1-\frac{2}{3} \hat{k}^{2}\right)^{2}}\right),
$$

enabling us to write the right hand side of (2.10) solely in terms of $\hat{\mathcal{H}}$.

So far we have just repeated part of the analysis performed in [12] and [4]. To continue we use the expression (2.10) and plug that into equation (2.7) to find the following expression for $\left(\hat{\mathcal{H}}^{4}\right)^{\hat{a} \hat{b}}$

$$
\left(\hat{\mathcal{H}}^{4}\right)^{\hat{a} \hat{b}}=\frac{2}{3} \hat{\mathcal{H}}^{2}\left[\hat{\eta}^{\hat{a} \hat{b}}+\frac{1}{2}\left(\hat{\mathcal{H}}^{2}\right)^{\hat{a} \hat{b}}\right] .
$$


Tracing equation (2.12) we obtain ${ }^{3}$

$$
\frac{1}{4} \hat{\mathcal{H}}^{4}=\hat{\mathcal{H}}^{2}\left(1+\frac{1}{12} \hat{\mathcal{H}}^{2}\right)
$$

It should be possible to deduce this constraint from the nonlinear self-duality equation (2.3) directly but we expect that to be more elaborate. From now on we will no longer use the (unphysical) field $\hat{h}$ (and the tensors that depend on it).

Using (2.12) one can easily verify that the inverse of $\hat{C}^{\hat{a} \hat{b}}$ is given by

$$
\left(\hat{C}^{-1}\right)_{\hat{a} \hat{b}}=\frac{1}{K}\left[\hat{\eta}_{\hat{a} \hat{b}}+\frac{1}{4}\left(\hat{\mathcal{H}}^{2}\right)_{\hat{a} \hat{b}}\right]
$$

We note that the traces of $\hat{C}$ and its inverse are both equal to $6 \sqrt{1+\frac{1}{24} \hat{\mathcal{H}}^{2}}$ and that we have, according to [4], the remarkable identity $\operatorname{det}\left(C^{-1}\right)_{a b}=\operatorname{det} g_{a b}$.

We define the open membrane co-metric as

$$
\hat{G}_{O M}^{\hat{a} \hat{b}}=z \hat{C}^{\hat{a} \hat{b}}
$$

and it will be our goal in the next section to determine the conformal factor $z$ by performing the double dimensional reduction.

\section{The open membrane metric on the circle}

First of all we split the $D=6$ indices into $\hat{a}=(a, y)$ where $x^{y}$ is a compact direction in the worldvolume of the M5-brane and identify the $D=5$ (dimensionless) 2-form and 3 -form fields as follows

$$
\begin{aligned}
\hat{\mathcal{H}}_{a b y} & \equiv \mathcal{F}_{a b} \\
\hat{\mathcal{H}}_{a b c} & \equiv \mathcal{H}_{a b c}
\end{aligned}
$$

As a consequence of the nonlinear self-duality equation in $D=6(2.3)$ the 3 -form $\mathcal{H}$ and the 2 -form $\mathcal{F}$ are related through a set of nonlinear duality equations given by

$$
\begin{aligned}
C_{a}{ }^{d} \mathcal{H}_{d b c}+C_{a}{ }^{y} \mathcal{F}_{b c} & =\frac{1}{2} \epsilon_{a b c d e} \mathcal{F}^{d e} \\
C_{y}{ }^{d} \mathcal{H}_{d a b}+C_{y}{ }^{y} \mathcal{F}_{a b} & =-\frac{1}{3 !} \epsilon_{a b d e f} \mathcal{H}^{\text {def }} \\
C_{a}{ }^{d} \mathcal{F}_{d b} & =-\frac{1}{3 !} \epsilon_{\text {abdef }} \mathcal{H}^{\text {def }}
\end{aligned}
$$

\footnotetext{
${ }^{3}$ Note that we will write $\left(\hat{\mathcal{H}}^{2}\right)^{2}$ to distinguish it from $\hat{\mathcal{H}}^{4}$, where the first expression should be understood in matrix notation as $\left(\operatorname{Tr}\left(\hat{\mathcal{H}}^{2}\right)^{\hat{a} \hat{b}}\right)^{2}$ and the second as $\operatorname{Tr}\left(\hat{\mathcal{H}}^{4}\right)^{\hat{a} \hat{b}}$.
} 
where the different components of $\hat{C}$ are

$$
\begin{aligned}
C^{a b} & \equiv \hat{C}^{a b}=\frac{1}{K}\left[\left(1+\frac{1}{12} \mathcal{H}^{2}+\frac{1}{4} \mathcal{F}^{2}\right) \eta^{a b}-\frac{1}{4}\left(\mathcal{H}^{2}\right)^{a b}-\frac{1}{2}\left(\mathcal{F}^{2}\right)^{a b}\right] \\
C^{a y} & =\frac{-1}{4 K} \mathcal{H}^{a}{ }_{c d} \mathcal{F}^{c d} \\
C^{y y} & =\frac{1}{K}\left(1+\frac{1}{12} \mathcal{H}^{2}\right) \eta^{y y} .
\end{aligned}
$$

In these expressions we used that $\hat{\mathcal{H}}^{2}=\mathcal{H}^{2}+3 \mathcal{F}^{2}$ and $\left(\hat{\mathcal{H}}^{2}\right)^{a b}=\left(\mathcal{H}^{2}\right)^{a b}+2\left(\mathcal{F}^{2}\right)^{a b} 4$.

Our goal is to express $C^{a b}$ and $C^{y y}$ solely in terms of $\mathcal{F}$, using the set of nonlinear duality equations $(3.2,3.3,3.4)$. Looking at these equations it is clear that this is not an easy problem. Instead of solving the equations (3.2, 3.3, 3.4) one could also look at the reduced expression for $\left(\hat{\mathcal{H}}^{4}\right)^{\hat{a} \hat{b}}(2.12)$, giving us the following set of equations

$$
\begin{gathered}
\left(\left(\mathcal{H}^{2}\right)^{a}{ }_{c}+2\left(\mathcal{F}^{2}\right)^{a}{ }_{c}\right)\left(\left(\mathcal{H}^{2}\right)^{c b}+2\left(\mathcal{F}^{2}\right)^{c b}\right)+\left(\mathcal{H}^{a c d} \mathcal{F}_{c d}\right)\left(\mathcal{H}^{b k l} \mathcal{F}_{k l}\right) \\
=\frac{2}{3}\left(\mathcal{H}^{2}+3 \mathcal{F}^{2}\right)\left(\eta^{a b}+\left(\mathcal{F}^{2}\right)^{a b}+\frac{1}{2}\left(\mathcal{H}^{2}\right)^{a b}\right) \\
{\left[\frac{1}{3} \mathcal{H}^{2} \eta^{a c}-\left(\left(\mathcal{H}^{2}\right)^{a c}+2\left(\mathcal{F}^{2}\right)^{a c}\right)\right] \mathcal{H}_{c m n} \mathcal{F}^{m n}=0} \\
\left(\mathcal{H}^{c k l} \mathcal{F}_{k l}\right)\left(\mathcal{H}_{c m n} \mathcal{F}^{m n}\right)-\frac{2}{3}\left(\mathcal{H}^{2}+3 \mathcal{F}^{2}\right)-\frac{1}{3} \mathcal{H}^{2} \mathcal{F}^{2}=0
\end{gathered}
$$

The solution to these equations can be found in the most general case [13], but in this paper we will find it useful to simplify matters considerably by insisting that the off-diagonal terms in the compact direction of the M5-brane Boillat co-metric vanish, i.e.

$$
V_{c} \equiv \mathcal{H}_{c m n} \mathcal{F}^{m n}=0
$$

This simplifies the equations considerably and we will proceed by focusing our attention on the duality equation (3.3), where the term containing $C_{y}{ }^{d}$ now vanishes. Multiplying that equation with $C_{y}^{y} \mathcal{F}^{a c}$ one finds the following useful relations (where the second one is obtained by tracing the first one ${ }^{5}$ )

$$
\begin{aligned}
\left(\mathcal{H}^{2}\right)^{a b} & =\frac{1}{1+\frac{1}{2} \mathcal{F}^{2}}\left(2\left(\mathcal{F}^{2}\right)^{a b}-\mathcal{F}^{2} \eta^{a b}\right) \\
\mathcal{H}^{2} & =\frac{-3 \mathcal{F}^{2}}{1+\frac{1}{2} \mathcal{F}^{2}} .
\end{aligned}
$$

\footnotetext{
${ }^{4}$ We note that our definition of $\left(\mathcal{F}^{2}\right)^{a b}$ differs by a minus sign with conventional matrix multiplication.

${ }^{5}$ Actually, using (3.11), the second equation can also be deduced directly from (3.10).
} 
This is all we need to write $C^{a b}$ and $C^{y y}$ strictly in terms of $\mathcal{F}$. As it turns out, we can also find a constraint on $\left(\mathcal{F}^{4}\right)^{a b}$. Using both results (3.12, 3.13) and plugging them into equation $(3.8)$ we find a surprisingly simple expression for $\left(\mathcal{F}^{4}\right)^{a b}$

$$
\left(\mathcal{F}^{4}\right)^{a b}=\frac{1}{2} \mathcal{F}^{2}\left(\mathcal{F}^{2}\right)^{a b}
$$

An analysis of this equation in terms of the eigenvalues of $\mathcal{F}^{a b}$ quickly reveals that our solutions for $\mathcal{F}^{a b}$ are restricted to rank 2 only. This is a direct consequence of the constraint (3.11) we imposed in order to simplify our analysis.

We are now ready to express $C^{a b}$ and $C^{y y}$ in terms of $\mathcal{F}$ only. We find

$$
\begin{aligned}
C^{a b} & =\frac{1}{\sqrt{1+\frac{1}{2} \mathcal{F}^{2}}}\left(\left(1+\frac{1}{2} \mathcal{F}^{2}\right) \eta^{a b}-\left(\mathcal{F}^{2}\right)^{a b}\right) \\
C^{y y} & =\frac{\eta^{y y}}{\sqrt{1+\frac{1}{2} \mathcal{F}^{2}}}
\end{aligned}
$$

and the inverse of $C^{a b}$, using (3.14),

$$
\left(C^{-1}\right)_{a b}=\frac{1}{\sqrt{1+\frac{1}{2} \mathcal{F}^{2}}}\left(\eta_{a b}+\left(\mathcal{F}^{2}\right)_{a b}\right)
$$

We note that by imposing that the off-diagonal components $C^{a y}$ vanish (3.11), we would have found the same result by reducing the inverse M5-brane Boillat metric (instead of reducing the M5-brane Boillat co-metric). This is no longer true when we would not have imposed this constraint.

The symmetric tensor $\left(C^{-1}\right)_{a b}$ should be equal to the Boillat metric. At first sight it looks like there is a discrepancy with the result obtained in 4 | where the conformal factor is equal to the inverse of $\sqrt{-\operatorname{det}\left(\eta_{a b}+\mathcal{F}_{a b}\right)}$ instead of $\sqrt{1+\frac{1}{2} \mathcal{F}^{2}}$. However, the 5 -dimensional determinant $-\operatorname{det}\left(\eta_{a b}+\mathcal{F}_{a b}\right)$ can be worked out to be equal to

$$
-\operatorname{det}\left(\eta_{a b}+\mathcal{F}_{a b}\right)=1+\frac{1}{2} \mathcal{F}^{2}+\frac{1}{8}\left(\mathcal{F}^{2}\right)^{2}-\frac{1}{4} \mathcal{F}^{4}
$$

Tracing the equation (3.14) it is clear that the last two terms in this expression of the determinant cancel each other and the right-hand side of 3.18 reduces to $1+\frac{1}{2} \mathcal{F}^{2}$. So we conclude that we end up with the expected result, that agrees with [1, 12, using a procedure in which we have restricted ourselves to consider rank $2 \mathcal{F}$ solutions only by imposing the (consistent) constraint $V_{c}=0$ (3.11).

Our next goal is to determine the conformal factor $z$ (2.15). As discussed in [9, 10], the relation between the 6-dimensional OM-theory parameter $\ell_{g}$ and the 5-dimensional 
NCOS parameters $\alpha_{o s}, \lambda_{o s}$, after the decoupling limit, mimics the bulk relations between M-theory and IIA string theory. An important assumption we will make is that these relations continue to hold beyond the decoupling limit. As a result, in analogy with the bulk relation $\eta^{y y} \equiv g_{s}^{-\frac{4}{3}}$, we will define the open string coupling constant $\lambda_{o s}$ as follows

$$
\lambda_{o s}^{-\frac{4}{3}} \equiv G_{O M}^{y y}=z C^{y y}=\frac{z g_{s}^{-\frac{4}{3}}}{\sqrt{1+\frac{1}{2} \mathcal{F}^{2}}} .
$$

We can now fix $z$ by demanding this expression to be equal to the Seiberg-Witten one [1] (replacing the determinant with our result $1+\frac{1}{2} \mathcal{F}^{2}$ )

$$
\lambda_{o s}=g_{s} \sqrt{1+\frac{1}{2} \mathcal{F}^{2}} .
$$

This determines $z$ to be equal to

$$
z=\left(1+\frac{1}{2} \mathcal{F}^{2}\right)^{-\frac{1}{6}}
$$

The next thing we should show is that upon including $g_{s}$ 's everywhere in our expressions for $C^{a b}$ we precisely find the open string metric and the open string coupling upon reduction of the open membrane metric. First of all we define the open membrane metric and the open string metric to be related in the following way (analogous to the well-known bulk relation $\left.\eta_{a b}=g_{s}^{-2 / 3} \eta_{a b}^{(s)}\right)$

$$
\left(G_{O M}^{-1}\right)_{a b} \equiv z^{-1}\left(C^{-1}\right)_{a b} \equiv \lambda_{o s}^{-\frac{2}{3}}\left(G_{O s}^{-1}\right)_{a b}
$$

Using this definition, plugging in our expressions for $z$ and $\lambda_{o s}$ we indeed find precisely the open string metric

$$
\left(G_{o s}^{-1}\right)_{a b}=\eta_{a b}^{(s)}+\left(\mathcal{F}^{2}\right)_{a b},
$$

where $\left(\mathcal{F}^{2}\right)_{a b}$ is now defined with respect to the string frame metric, i.e. $\left(\mathcal{F}^{2}\right)_{a b}=$ $\mathcal{F}_{a c} \eta_{(s)}^{c d} \mathcal{F}_{b d}$. We have now successfully shown that the open membrane metric, with the previously unknown conformal factor $z$ now determined, reduces to the Seiberg-Witten expressions for the open string metric and the open string coupling. We note that our procedure that lead to this result was restricted to rank $2 \mathcal{F}$ only and assumed reduction ansatze analogous to the bulk.

The only thing left to do is to rewrite this conformal factor $z(3.21)$ in terms of $\hat{\mathcal{H}}$, using (3.13) and the fact that $\hat{\mathcal{H}}^{2}=\mathcal{H}^{2}+3 \mathcal{F}^{2}$. It turns out to be useful to write this expression in terms of $K(2.2)$ and we find

$$
z=\left(\left(2 K^{2}-1\right) \pm 2 K^{2} \sqrt{1-K^{-2}}\right)^{-\frac{1}{6}}
$$


Because the relation between $\hat{\mathcal{H}}^{2}$ and $\mathcal{F}^{2}$ is quadratic there is a sign ambiguity in this expression.

The sign ambiguity can be fixed by performing the following consistency check. Our result for the conformal factor should reproduce the result reported in [10] that the conformal factor scales as $\left(\frac{\ell_{p}}{\ell_{g}}\right)^{2}$ in the OM-theory decoupling limit. One can check that in the OM-theory limit $K^{2}$ scales as

$$
K^{2}=1+\frac{1}{24} \hat{\mathcal{H}}^{2} \sim\left(\frac{\ell_{g}}{\ell_{p}}\right)^{3} .
$$

As it turns out, only when we choose the $(-) \operatorname{sign}$ in the expression for $z$ (3.24), due to some crucial cancellations (expanding the square root to second order), the conformal factor precisely scales as

$$
\frac{\left[\left(2 K^{2}-1\right)-2 K^{2} \sqrt{1-K^{-2}}\right]^{\frac{1}{6}}}{K} \sim\left(K^{2}\right)^{-\frac{2}{3}}=\left(\frac{\ell_{p}}{\ell_{g}}\right)^{2}
$$

therefore reproducing the result of [10]. Taking the $(+)$ sign however, results in a diverging open membrane metric with respect to the Planck length $\ell_{p}$ (the conformal factor is proportional to $\frac{\ell_{p}}{\ell_{g}}$ to leading order) in the OM-theory decoupling limit. So, finally, after getting rid of the sign ambiguity to agree with the expected behavior in the decoupling limit, the open membrane metric is determined to be equal to

$$
\left(G_{O M}^{-1}\right)_{a b}=\frac{\left(\left(2 K^{2}-1\right)-2 K^{2} \sqrt{1-K^{-2}}\right)^{\frac{1}{6}}}{K}\left[\hat{\eta}_{\hat{a} \hat{b}}+\frac{1}{4}\left(\hat{\mathcal{H}}^{2}\right)_{\hat{a} \hat{b}}\right] .
$$

\section{The open membrane metric on the torus}

Our results support the idea that the open membrane metric can be understood, upon reduction, as providing the geometric origin of the open string (or more generally, open brane) moduli, independent of whether the M5-brane or D-brane is decoupled from the bulk or not. This also implies the existence of an $S L(2, Z)$ generalization of the open string coupling constant, i.e. a complex open string coupling constant, which should equal the modular parameter of the (OM) torus, analogous to what happens when wrapping M-theory on a torus.

We will consider $z^{i}$, with $i=4,5$, to be the coordinates on the torus and $x^{a}$, with $a=0,1, \ldots, 3$ as the (directly reduced) D3-brane coordinates. From the start we will assume that the off-diagonal open membrane (co-) metric components $\hat{G}^{a i}$ vanish, again 
effectively restricting us to rank 2 solutions. For our purposes here we will also assume that after reduction on the $T^{2}$ we will be left with 2 -forms only ${ }^{6}$. So we define

$$
\begin{aligned}
\hat{\mathcal{H}}^{a b i} & \equiv \mathcal{F}^{a b, i} \\
\hat{\mathcal{H}}^{a i j} & \equiv \mathcal{V}^{a} \equiv 0 \\
\hat{\mathcal{H}}^{a b c} & \equiv \mathcal{H}^{a b c} \equiv 0 .
\end{aligned}
$$

Because we restricted the fields $\mathcal{H}$ and $\mathcal{V}$ to be zero, we can write

$$
\left(\hat{\mathcal{H}}^{2}\right)^{a b}=2\left(\mathcal{F}^{i} \zeta_{i j} \mathcal{F}^{j}\right)^{a b}
$$

and

$$
\hat{\mathcal{H}}^{2}=3 \mathcal{F}^{i} \zeta_{i j} \mathcal{F}^{j}
$$

where $\zeta_{i j}$ is the metric on the torus (it is understood that the absence of $D=4$ spacetime indices means they are summed over). Obviously these expressions are $S L(2, R)$ invariant. Again, as a consequence of the nonlinear self-duality equation on the M5brane, the doublet of two-forms on the D3-brane has to satisfy the following set of (self-) duality equations

$$
\begin{aligned}
C^{i j} \mathcal{F}_{a b, j} & =\frac{1}{2} \epsilon_{\text {abef }}\left(\epsilon^{i j} \mathcal{F}^{e f}{ }_{j}\right) \\
C^{a d} \mathcal{F}_{d b, i} & =\frac{1}{2} \epsilon_{a b e f}\left(\epsilon_{i j} \mathcal{F}^{e f, j}\right),
\end{aligned}
$$

where the tensors $C$ are now given by

$$
\begin{aligned}
C^{i j} & =\frac{1}{K}\left[\left(1+\frac{1}{4} \mathcal{F}^{k} \zeta_{k l} \mathcal{F}^{l}\right) \zeta^{i j}-\frac{1}{4}\left(\mathcal{F}^{2}\right)^{i j}\right] \\
C^{a b} & =\frac{1}{K}\left[\left(1+\frac{1}{4} \mathcal{F}^{k} \zeta_{k l} \mathcal{F}^{l}\right) \eta^{a b}-\frac{1}{2}\left(\mathcal{F}^{k} \zeta_{k l} \mathcal{F}^{l}\right)^{a b}\right] .
\end{aligned}
$$

The reduction of equation (2.12) gives the following relations for $\left(\mathcal{F}^{2}\right)^{i k} \zeta_{k l}\left(\mathcal{F}^{2}\right)^{j k}$ and $\left(\left(\mathcal{F}^{k} \zeta_{k l} \mathcal{F}^{l}\right)^{2}\right)^{a b}$

$$
\begin{aligned}
& \left(\mathcal{F}^{2}\right)^{i k} \zeta_{k l}\left(\mathcal{F}^{2}\right)^{j l}=2\left(\mathcal{F}^{k} \zeta_{k l} \mathcal{F}^{l}\right)\left[\zeta^{i j}+\frac{1}{2}\left(\mathcal{F}^{2}\right)^{i j}\right] \\
& \left(\left(\mathcal{F}^{k} \zeta_{k l} \mathcal{F}^{l}\right)^{2}\right)^{a b}=\frac{1}{2}\left(\mathcal{F}^{k} \zeta_{k l} \mathcal{F}^{l}\right)\left[\eta^{a b}+\left(\mathcal{F}^{k} \zeta_{k l} \mathcal{F}^{l}\right)^{a b}\right]
\end{aligned}
$$

\footnotetext{
${ }^{6}$ One can check that after imposing $\hat{G}_{a i}=0$ the duality equations relating the 2 -forms 'decouple' from the duality equations relating the 1 - and 3-forms, allowing for a consistent truncation with vanishing 1 - and 3 -forms.
} 
which are useful for checking that the inverses of $C$ on the torus and the D3-brane are given by

$$
\begin{aligned}
\left(C^{-1}\right)_{i j} & =\frac{1}{K}\left[\zeta_{i j}+\frac{1}{4}\left(\mathcal{F}^{2}\right)_{i j}\right] \\
\left(C^{-1}\right)_{a b} & =\frac{1}{K}\left[\eta_{a b}+\frac{1}{2}\left(\mathcal{F}^{k} \zeta_{k l} \mathcal{F}^{l}\right)_{a b}\right] .
\end{aligned}
$$

Previously, when reducing the M5-brane on a circle we used the duality equations to rewrite everything in terms of $\mathcal{F}$ only and we obtained the expected Seiberg-Witten results. However, in this case the set of duality equations generically relate the $S L(2, R)$ doublet $\mathcal{F}^{i}$ to the doublet $\mathcal{F}^{i}$ in a complicated (intertwined) way, i.e. it is more like a self-duality equation. Another way of saying this is that generically both electric and magnetic components of both field strengths in the doublet have to be turned on to satisfy the set of duality equations (4.4) and (4.5). The question arises as to how we can obtain the Seiberg-Witten results for the D3-brane, which should still be valid when only one of the 2 -forms in the doublet is turned on ${ }^{7}$.

The answer is that in the special case where the off-diagonal components of $C^{i j}$ vanish, the set of duality equations allows for solutions with only one of the 2-forms turned on in a particular direction, i.e. the set of self-duality equations reduces to a set of ordinary (nonlinear) duality equations relating one of the 2 -forms in the doublet to the other one. This enables us to follow the same procedure as before, solving for just one of the 2-forms in the doublet and we will check that this indeed gives the expected result for the open string metric and coupling constant. Another way to understand this is that in a generic $S L(2, R)$ basis we are clearly forced to use both 2 -forms and the result for the open membrane metric is proportional to (4.10), whereas in the special case we should be able to rewrite (4.10) in terms of just one of the 2-forms and it is only then that we will find the Seiberg-Witten results. It must be clear that on the D3-brane it is always possible to find such an $S L(2, R)$ basis $^{8}$, at the cost of giving up manifest $S L(2, R)$ invariance.

After clearing that up we now want to rewrite our results (4.10) and (4.11) in terms of the appropriate open string quantities, the open string ("Einstein") metric $G_{\text {osE }}^{-1}$ and the complex open string coupling constant $T=X+i \lambda_{o s}^{-1}$. First of all we make the following standard identifications of the bulk quantities $\left(\tau=\chi+i g_{s}^{-1}\right)$

$$
\eta_{a b}=\frac{1}{\sqrt{A_{T^{2}}^{(\text {bulk })}}} \eta_{a b}^{(E)}
$$

\footnotetext{
${ }^{7}$ To be more precise, we need the off-diagonal components of $C^{i j}$ to vanish. As we will see, this means that the bulk axion has to vanish as well.

${ }^{8}$ Upon considering $S L(2, Z)$ this is no longer necessarily true, in that case one needs a rational axion to be able to rotate to a frame in which the axion vanishes.
} 


$$
\begin{aligned}
A_{T^{2}}^{(\mathrm{bulk})} & =\sqrt{\operatorname{det} \zeta_{i j}}=\sqrt{\zeta_{44} \zeta_{55}-\zeta_{45}^{2}} \\
\chi & =-\frac{\zeta_{45}}{\zeta_{44}} \\
g_{s}^{-2} & =\frac{\zeta_{55}-\frac{\zeta_{45}^{2}}{\zeta_{44}}}{\zeta_{44}} .
\end{aligned}
$$

Together with (4.1), this enables us to write the $S L(2, R)$ invariant quantity $\left(\mathcal{F}^{i} \zeta_{i j} \mathcal{F}^{j}\right)$ as follows

$$
\left(\mathcal{F}^{i} \zeta_{i j} \mathcal{F}^{j}\right)=g_{s}\left(\mathcal{F}^{4}-\chi \mathcal{F}^{5}\right)^{2}+g_{s}^{-1}\left(\mathcal{F}^{5}\right)^{2}
$$

which is invariant under the following $S L(2, R)$ transformations

$$
\begin{aligned}
\tau & \rightarrow \frac{a \tau+b}{c \tau+d} \\
\left(\begin{array}{l}
\mathcal{F}^{4} \\
\mathcal{F}^{5}
\end{array}\right) & \rightarrow\left(\begin{array}{ll}
a & b \\
c & d
\end{array}\right)\left(\begin{array}{l}
\mathcal{F}^{4} \\
\mathcal{F}^{5}
\end{array}\right)
\end{aligned}
$$

with $a d-b c=1$. We note that when calculating $\left(\mathcal{F}^{i} \zeta_{i j} \mathcal{F}^{j}\right)_{a b}$, because we defined the two-forms contravariantly (4.1), we obtain an extra factor $\left(A_{T^{2}}^{(b u l k)}\right)^{-\frac{1}{2}}$ as compared to equation (4.16).

The next step is to define the corresponding open string quantities on the D3-brane in an analogous way using the open membrane metric (3.27). So we define

$$
\begin{aligned}
\left(G_{O M}^{-1}\right)_{a b} & \equiv \frac{1}{\sqrt{A_{T^{2}}^{(\mathrm{OM})}}\left(G_{O S E}^{-1}\right)_{a b}} \\
A_{T^{2}}^{(\mathrm{OM})} & =\sqrt{\operatorname{det}\left(G_{O M}^{-1}\right)_{i j}}=\sqrt{\left(G_{O M}^{-1}\right)_{44}\left(G_{O M}^{-1}\right)_{55}-\left(G_{O M}^{-1}\right)_{45}^{2}} \\
X & \equiv-\frac{\left(G_{O M}^{-1}\right)_{45}}{\left(G_{O M}^{-1}\right)_{44}} \\
\lambda_{O s}^{-2} & \equiv \frac{\left(G_{O M}^{-1}\right)_{55}-\frac{\left(G_{O M}^{-1}\right)_{45}^{2}}{\left(G_{O M}^{-1}\right)_{44}}}{\left(G_{O M}^{-1}\right)_{44}} .
\end{aligned}
$$

Using these definitions and (4.1), we find the following results for the D3-brane open string quantities expressed in terms of the appropriate bulk quantities (4.12)-(4.15) ${ }^{9}$

$$
\left(G_{\text {osE }}^{-1}\right)_{a b}=\frac{1}{z K} \sqrt{\frac{A_{T^{2}}^{(\mathrm{OM})}}{A_{T^{2}}^{(\mathrm{bulk})}}}\left[\eta_{a b}^{(E)}+\frac{1}{2}\left(g_{s}\left(\mathcal{F}^{4}-\chi \mathcal{F}^{5}\right)^{2}+g_{s}^{-1}\left(\mathcal{F}^{5}\right)^{2}\right)_{a b}\right]
$$

\footnotetext{
${ }^{9}$ We apologize for using the numbers 4 and 5 to denote the different components of the $S L(2, R)$ vector, which should not be confused with taking fourth or fifth powers of $\mathcal{F}$.
} 


$$
\begin{aligned}
X & =\frac{\chi+\frac{1}{4} g_{s}\left(\chi \mathcal{F}^{4}-|\tau|^{2} \mathcal{F}^{5}\right)_{a b}\left(\mathcal{F}^{4}-\chi \mathcal{F}^{5}\right)^{a b}}{1+\frac{1}{4} g_{s}\left(\mathcal{F}^{4}-\chi^{\mathcal{F}^{5}}\right)^{2}} \\
\lambda_{o s}^{-2} & =\frac{|\tau|^{2}+\frac{1}{4} g_{s}\left(\chi \mathcal{F}^{4}-|\tau|^{2} \mathcal{F}^{5}\right)^{2}}{1+\frac{1}{4} g_{s}\left(\mathcal{F}^{4}-\chi \mathcal{F}^{5}\right)^{2}}-X^{2}
\end{aligned}
$$

where it should be understood that all contractions of $D=4$ indices are now taken with respect to the Einstein frame metric $\eta_{a b}^{(E)}$ and where $z$ is the conformal factor that was determined in the previous section (3.24). For completeness we should also give the expressions for $K^{2}(2.2)$ and $A_{T^{2}}^{(\mathrm{OM})}$ (in terms of bulk quantities)

$$
\begin{aligned}
K^{2} & =1+\frac{1}{8}\left(g_{s}\left(\mathcal{F}^{4}-\chi \mathcal{F}^{5}\right)^{2}+g_{s}^{-1}\left(\mathcal{F}^{5}\right)^{2}\right) \\
A_{T^{2}}^{(\mathrm{OM})} & =\left(G_{O M}^{-1}\right)_{44} \lambda_{o s}^{-1}=\frac{A_{T^{2}}^{(\text {bulk })} g_{s}}{z K}\left(1+\frac{1}{4} g_{s}\left(\mathcal{F}^{4}-\chi \mathcal{F}^{5}\right)^{2}\right) \lambda_{o s}^{-1} .
\end{aligned}
$$

As a first check that these expressions are correct we observe that S-duality transformations of bulk quantities induce S-duality transformations on the D3-brane. One can check that upon the S-duality transformation

$$
\mathcal{F}^{4} \leftrightarrow \mathcal{F}^{5}, \quad \tau \rightarrow \frac{1}{\tau}
$$

one induces the following S-duality transformation on the open string modular parameter $T$

$$
T \rightarrow \frac{1}{T}
$$

Another straightforward check is to consider shift transformations, i.e.

$$
\mathcal{F}^{4} \rightarrow \mathcal{F}^{4}+b \mathcal{F}^{5}, \quad \mathcal{F}^{5} \rightarrow \mathcal{F}^{5}, \quad \tau \rightarrow \tau+b
$$

Although perhaps not immediately obvious these transformations indeed induce shift transformations on the open string modular parameter $T$, i.e.

$$
T \rightarrow T+b
$$

Based on these explicit checks we are therefore confident that the full group of $S L(2, R)$ transformations is indeed induced from the closed string modular parameter $\tau$ onto the open string modular parameter $T$ (also transforming the $S L(2, R)$ vector $\mathcal{F}^{i}$ ), as it should.

We now want to check that when we take $\chi=0$ and $\left(\mathcal{F}^{4}\right)_{a b}\left(\mathcal{F}^{5}\right)^{a b}=0$ (giving $X=0$ and necessarily giving up manifest $S L(2, R)$ invariance) we obtain the SeibergWitten results. This should involve solving $\mathcal{F}^{4}$ in terms of $\mathcal{F}^{5}$ with the help of the 
duality equations (4.4) (solving for $\mathcal{F}^{5}$ we expect to find the S-dual result). Setting $\chi=0$ in (4.23)-(4.25) gives us

$$
\begin{aligned}
& \left(G_{\text {os } E}^{-1}\right)_{a b}=\frac{1}{z K} \sqrt{\frac{A_{T^{2}}^{(\mathrm{OM})}}{A_{T^{2}}^{(\mathrm{bulk})}}}\left[\eta_{a b}^{(E)}+\frac{1}{2}\left(g_{s}\left(\mathcal{F}^{4}\right)^{2}+g_{s}^{-1}\left(\mathcal{F}^{5}\right)^{2}\right)_{a b}\right] \\
& \lambda_{o s}^{-2}=g_{s}^{-2}\left(\frac{1+\frac{1}{4} g_{s}^{-1}\left(\mathcal{F}^{5}\right)^{2}}{1+\frac{1}{4} g_{s}\left(\mathcal{F}^{4}\right)^{2}}\right) \text {. }
\end{aligned}
$$

When $X=0$ (giving us only diagonal entries in $C^{i j}$ ) we can now use the duality equation (4.4) and/or equation (4.8) to write $\mathcal{F}^{5}$ in terms of $\mathcal{F}^{4}$. The appropriate relations that can be deduced are

$$
\begin{aligned}
g_{s}^{-1}\left(\mathcal{F}^{5}\right)_{a b}^{2} & =\frac{1}{1+\frac{1}{2} g_{s}\left(\mathcal{F}^{4}\right)^{2}}\left[g_{s}\left(\mathcal{F}^{4}\right)_{a b}^{2}-\frac{1}{2} g_{s}\left(\mathcal{F}^{4}\right)^{2} \eta_{a b}^{(E)}\right] \\
g_{s}^{-1}\left(\mathcal{F}^{5}\right)^{2} & =\frac{-g_{s}\left(\mathcal{F}^{4}\right)^{2}}{1+\frac{1}{2} g_{s}\left(\mathcal{F}^{4}\right)^{2}} .
\end{aligned}
$$

We note that by performing an S-duality transformation $g_{s}\left(\mathcal{F}^{4}\right)^{2} \rightarrow g_{s}^{-1}\left(\mathcal{F}^{5}\right)^{2}$ we obtain the other set of equations (which are of course redundant because they follow uniquely from (4.34) and (4.35)). These equations can first of all be used to find the following expression for the conformal factor in $\left(G_{o s E}^{-1}\right)_{a b}(4.23)$ (also using (3.24), (4.26) and (4.27))

$$
\frac{1}{z K} \sqrt{\frac{A_{T^{2}}^{(\mathrm{OM})}}{A_{T^{2}}^{(\mathrm{bulk})}}}=\frac{\left(1+\frac{1}{2} g_{s}\left(\mathcal{F}^{4}\right)^{2}\right)^{\frac{3}{4}}}{1+\frac{1}{4} g_{s}\left(\mathcal{F}^{4}\right)^{2}} .
$$

Plugging these expressions (4.34)-(4.36) into the D3-brane open string metric (4.32) and open string coupling (4.33) we find the following results

$$
\begin{aligned}
\left(G_{o s E}^{-1}\right)_{a b} & =\left(1+\frac{1}{2} g_{s}\left(\mathcal{F}^{4}\right)^{2}\right)^{-\frac{1}{4}}\left[\eta_{a b}^{(E)}+g_{s}\left(\mathcal{F}^{4}\right)_{a b}^{2}\right] \\
\lambda_{o s} & =g_{s} \sqrt{1+\frac{1}{2} g_{s}\left(\mathcal{F}^{4}\right)^{2}}
\end{aligned}
$$

We should keep in mind that until now we have been using a contravariant definition of the 2-form field strengths (4.1), which is not standard. To transform to the standard covariant definition one actually needs to include a factor of $g_{s}^{-2}=\left(A_{T^{2}}^{(b u l k)}\right)^{2}\left(\zeta^{44}\right)^{2}$ in all $\left(\mathcal{F}^{4}\right)^{2}$ terms. Doing this and realizing that we should now transform to the string frame metric, using $\eta_{a b}^{(s)}=g_{s}^{\frac{1}{2}} \eta_{a b}^{(E)}$ and defining analogously

$$
\left(G_{o s}^{-1}\right)_{a b} \equiv \lambda_{o s}^{\frac{1}{2}}\left(G_{o s E}^{-1}\right)_{a b}
$$


we finally obtain the open string metric and coupling, as promised,

$$
\begin{aligned}
\left(G_{o s}^{-1}\right)_{a b} & =\eta_{a b}^{(s)}+\left(\mathcal{F}_{4}\right)_{a b}^{2} \\
\lambda_{o s} & =g_{s} \sqrt{1+\frac{1}{2}\left(\mathcal{F}_{4}\right)^{2}} .
\end{aligned}
$$

As mentioned before, when solving in terms of $\mathcal{F}^{5}$ instead, we expect to find the S-dual result. We want to emphasize however that performing an S-duality transformation is definitely not the same as solving in terms of the other field in the doublet. Using the duality equations one interchanges electric and magnetic components, whereas the S-duality transformations do not interchange electric and magnetic components. The fact that we do find the S-dual result explains why on the D3-brane S-duality can be effectively represented by an interchange of electric and magnetic field strengths; starting with an electric $\mathcal{F}^{4}$, S-duality gives us an electric $\mathcal{F}^{5}$, but by using the duality equations we can relate this electric $\mathcal{F}^{5}$ to a magnetic $\mathcal{F}^{4}$ giving us an effective interchange of electric and magnetic components of $\mathcal{F}^{4}$ upon S-duality. Starting from (4.32) and $(4.33)$ and now solving for $\mathcal{F}^{5}$ we indeed find

$$
\begin{aligned}
\left(G_{o s}^{-1}\right)_{a b} & =\frac{\lambda_{o s}}{g_{s}}\left[\eta_{a b}^{(s)}+g_{s}^{2}\left(\mathcal{F}_{5}\right)_{a b}^{2}\right] \\
\lambda_{o s} & =\frac{g_{s}}{\sqrt{1+\frac{1}{2} g_{s}^{2}\left(\mathcal{F}_{5}\right)^{2}}}
\end{aligned}
$$

which can easily be checked to be equivalent to performing an S-duality transformation on (4.40) and (4.41) (remember that the string frame metric transforms as $\eta_{a b}^{(s)} \rightarrow \frac{\eta_{a b}^{(s)}}{g_{s}}$ ). It seems natural to relate the above result to an open D-string metric $\left(G_{o d}^{-1}\right)_{a b}$ and open D-string coupling, defined in the following way

$$
\left(G_{o d}^{-1}\right)_{a b} \equiv \frac{\left(G_{o s}^{-1}\right)_{a b}}{\lambda_{o s}}, \quad \lambda_{o d} \equiv \frac{1}{\lambda_{o s}}
$$

Also identifying $\frac{\eta^{(s)}}{g_{s}} \equiv \eta^{(d)}$ and rewriting the square of $\mathcal{F}^{5}$ with respect to this metric, we can rewrite the expressions (4.42) and (4.43) as

$$
\begin{aligned}
\left(G_{o d}^{-1}\right)_{a b} & =\eta_{a b}^{(d)}+\left(\mathcal{F}_{5}\right)_{a b}^{2} \\
\frac{1}{\lambda_{o d}} & =\lambda_{o s}=\frac{g_{s}}{\sqrt{1+\frac{1}{2}\left(\mathcal{F}_{5}\right)^{2}}}
\end{aligned}
$$

This metric can also be used to describe the same physics on the D3-brane, a priori there is no reason to prefer using the open string metric instead of the open D-string metric. Only when considering a particular limit of the closed string moduli will one 
create a distinction. To give a concrete example; it is not hard to show that the NCOS limit in (4.40) and (4.41) maps to the open D-string NCYM limit in (4.45) and (4.46), illustrating the fact that the NCOS theory can equivalently be described by an open D-string NCYM theory (and vice-versa of course) [23, 24].

Going back to our $S L(2, R)$ covariant expressions (4.23), (4.24) and (4.25) it seems natural to interpret these as a result of probing the D3-brane with open $(p, q)$ strings [25]. The existence of an OM-theory decoupling limit should at first sight guarantee the existence of a correspondingly well-defined decoupling limit for $(p, q)$ open strings ending on the D3-brane. Indeed, the situation is very similar to the M5-brane, because generically our doublet of 2-forms satisfies a nonlinear self-duality equation (4.5). This means we will have to consider constant 2-form doublet field strengths in all directions (electric and magnetic) on the D3-brane. It should be possible to deduce a parametrisation of these (constant flux) solutions by reducing the M5-brane parametrisation as given in [2], but we will not do so here [26]. Another observation strengthening our belief that the decoupling limit for $(p, q)$ strings is similar to the one on the M5-brane, is that in the OM-theory limit the conformal factor of the $(p, q)$ open string metric (4.23) scales as

$$
\frac{1}{z K} \sqrt{\frac{A_{T^{2}}^{(\mathrm{OM})}}{A_{T^{2}}^{(\mathrm{bulk})}}} \sim\left(\frac{l_{p}}{l_{g}}\right)^{3} \propto \frac{\alpha^{\prime}}{\alpha_{(e f f)}^{\prime}} .
$$

This means that in order to obtain a finite length scale $\alpha_{(e f f)}^{\prime}$ the other part of the open $(p, q)$ string metric (4.23) should be fixed in the decoupling limit, analogous to what happens in the OM-theory limit on the M5-brane.

\section{Summary and discussion}

For the readers convenience let us start this section by summarizing the main results presented in this paper. To fix the conformal factor of the open membrane metric we first of all assumed the following reduction ansatze (in analogy to the bulk) between the open membrane and open string moduli

$$
\begin{aligned}
\left(G_{O M}^{-1}\right)_{a b} & \equiv \lambda_{o s}^{-\frac{2}{3}}\left(G_{O S}^{-1}\right)_{a b} \\
\left(G_{O M}^{-1}\right)_{y y} & \equiv \lambda_{o s}^{\frac{4}{3}}
\end{aligned}
$$

Upon reduction we effectively restricted our attention to rank 2 solutions by imposing the (simplifying) constraint $\mathcal{H}^{a m n} \mathcal{F}_{m n}=0$. Using one of the two reduction ansatze fixes the conformal factor of the open membrane metric in order to reproduce one of the Seiberg-Witten results, the other definition then consistently reproduces the 
other Seiberg-Witten result. In the reduction procedure we made important use of the following relations, which are consequences of the (self-) duality equations and imposing $\mathcal{H}^{a m n} \mathcal{F}_{m n}=0$ upon reduction

$$
\begin{aligned}
\left(\hat{\mathcal{H}}^{4}\right)^{\hat{a} \hat{b}} & =\frac{2}{3} \hat{\mathcal{H}}^{2}\left[\hat{\eta}^{\hat{a} \hat{b}}+\frac{1}{2}\left(\hat{\mathcal{H}}^{2}\right)^{\hat{a} \hat{b}}\right] \\
\left(\mathcal{H}^{2}\right)^{a b} & =\frac{1}{1+\frac{1}{2} \mathcal{F}^{2}}\left(2\left(\mathcal{F}^{2}\right)^{a b}-\mathcal{F}^{2} \eta^{a b}\right) \\
\left(\mathcal{F}^{4}\right)^{a b} & =\frac{1}{2} \mathcal{F}^{2}\left(\mathcal{F}^{2}\right)^{a b},
\end{aligned}
$$

where the last constraint on $\left(\mathcal{F}^{4}\right)^{a b}$ implies that we have restricted ourselves to rank 2 solutions only and

$$
\sqrt{-\operatorname{det}\left(\eta_{a b}+\mathcal{F}_{a b}\right)}=\sqrt{1+\frac{1}{2} \mathcal{F}^{2}}
$$

Using all this information we showed that the following open membrane metric

$$
\left(G_{O M}^{-1}\right)_{a b}=\frac{\left(\left(2 K^{2}-1\right)-2 K^{2} \sqrt{1-K^{-2}}\right)^{\frac{1}{6}}}{K}\left[\hat{\eta}_{\hat{a} \hat{b}}+\frac{1}{4}\left(\hat{\mathcal{H}}^{2}\right)_{\hat{a} \hat{b}}\right]
$$

indeed scales as anticipated in [10] in the OM-theory decoupling limit and reproduces the open string metric and coupling as first given in [1] upon double dimensional reduction (for rank 2 solutions).

One thing to worry about is whether our solution for the conformal factor $z$ depends on our restriction to rank 2 solutions. Because from the point of view of the M5-brane the difference between rank 2 and rank 4 solutions is nothing but a trivial rotation, we would argue that our final result is independent of that restriction. However, it would be interesting and worthwhile to show this by actually performing the reduction in this more general (and more complicated) case and we hope to report on this (and some other issues) in the near future [26].

After fixing the conformal factor we performed a double dimensional reduction of the M5-brane on a 2-torus (again effectively restricting our attention to rank 2 solutions only) to obtain manifestly $S L(2, R)$ covariant results for the open string metric and complex open string coupling $T=X+i \lambda_{o s}$

$$
\begin{aligned}
& \left(G_{\text {osE }}^{-1}\right)_{a b}=\frac{1}{z K} \sqrt{\frac{A_{T^{2}}^{(\mathrm{OM})}}{A_{T^{2}}^{(\mathrm{bulk})}}}\left[\eta_{a b}^{(E)}+\frac{1}{2}\left(g_{s}\left(\mathcal{F}^{4}-\chi \mathcal{F}^{5}\right)^{2}+g_{s}^{-1}\left(\mathcal{F}^{5}\right)^{2}\right)_{a b}\right] \\
& X=\frac{\chi+\frac{1}{4} g_{s}\left(\chi \mathcal{F}^{4}-|\tau|^{2} \mathcal{F}^{5}\right)_{a b}\left(\mathcal{F}^{4}-\chi \mathcal{F}^{5}\right)^{a b}}{1+\frac{1}{4} g_{s}\left(\mathcal{F}^{4}-\chi \mathcal{F}^{5}\right)^{2}} \\
& \lambda_{o s}^{-2}=\frac{|\tau|^{2}+\frac{1}{4} g_{s}\left(\chi \mathcal{F}^{4}-|\tau|^{2} \mathcal{F}^{5}\right)^{2}}{1+\frac{1}{4} g_{s}\left(\mathcal{F}^{4}-\chi \mathcal{F}^{5}\right)^{2}}-X^{2} \text {. }
\end{aligned}
$$


We showed that S-duality and shift transformations of the bulk quantities induce Sduality and shift transformations on the D3-brane quantities, as they should. To reproduce the Seiberg-Witten NCOS metric and coupling we used the nonlinear duality equations on the D3-brane and we emphasized the important role played by the duality equations in the context of manifest $S L(2, R)$ invariance on the D3-brane. Here we would also like to anticipate the existence of an elegant manifestly $S L(2, R)$ covariant formulation of the (single) D3-brane equations of motion (and perhaps even an action) in terms of the $S L(2, R)$ invariant open string metric. The natural interpretation of our $S L(2, R)$ covariant results would be in terms of (non-commutative) $(p, q)$ open strings and we gave some (mainly OM-theory) arguments to suggest that a $(p, q)$ open string decoupling limit should exist.

One thing we did not consider in this paper is the non-commutativity parameter $\theta_{a b}$. We concentrated our attention on the open string metrics and coupling as they can be directly related to the reduced open membrane metric. As explained in [3], there does exist a nonlinear electrodynamics analogue of the non-commutativity parameter which is related to the dual Maxwell field. It would be interesting to find the expression for the non-commutativity parameter in our $S L(2, R)$ covariant case. Also, it would be very interesting to see if and how we can generalize this relation between the noncommutativity parameter and the dual Maxwell field to the M5-brane and the self-dual tensor multiplet. In [4] a 3-form $P$ was introduced on the M5-brane which could have all the properties we are looking for. We hope to report on this interesting possibility in a future publication 26].

As a consequence of quantum effects one expects the $S L(2, R)$ symmetry group to reduce to $S L(2, Z)$. One immediate consequence would be that only when the axion is rational can we $S L(2, Z)$ rotate into a frame in which the axion vanishes. The same is therefore true for the D3-brane (open string) axion $X$; it has to be rational in order to be able to $S L(2, Z)$ transform to a frame where $X$ vanishes ${ }^{10}$. This would imply that when $X$ is irrational one is forced to present the D3-brane theory in terms of an $S L(2, Z)$ doublet of field strengths. This is just an observation, it is not clear to us at this time whether this has any profound meaning.

There are many extensions of our work that one can think of. First of all, the precise decoupling limit describing the non-commutative $(p, q)$ open strings in this context still has to be worked out. Doing this is expected to connect to (some of) the results reported in [22], either by using a 'flat space' closed string moduli approach or by making use of holographic methods (using the dual supergravity background in order

\footnotetext{
${ }^{10}$ Similarly one needs a rational axion to be able to $S L(2, Z)$ rotate into a perturbative open string regime.
} 
to find a decoupling limit) 27]. A very concrete goal would for example be to obtain the $S L(2, R)$ invariant tension formula for the decoupled non-commutative open $(p, q)$ strings on the D3-brane. Another interesting project would be to see whether we can generalize our discussion of $S L(2, R)$ covariant moduli of open strings on the D3-brane to the worldvolume of $(m, n)$ five-branes in Type IIB theory [22]. This will inevitably introduce a 'little (closed) strings' sector into the theory and it would be interesting to see how this would affect our results. At first sight it seems like a good idea to take an OM-theory or M5-brane perspective again, because little strings can be naturally understood as open membranes wrapped around a compact direction transverse to the M5-brane (this idea was first exploited in [24]).

From another point of view it would be very nice if one could provide a more direct understanding of the open membrane metric. So instead of defining this object indirectly via string theory, we would prefer to understand the open membrane metric directly from an M-theory perspective. Quantizing the open membrane presumably has all the usual problems, so that does not seem to help us. A more fruitful, less ambitious, point of view seems to be the conformal relation of the open membrane metric to the M5-brane Boillat metric [4]. This relation in some sense leaves us with the 'smaller' problem of 'explaining' the (rather complicated) conformal factor of the open membrane metric (3.27) as compared to the M5-brane Boillat metric (where the M5-brane Boillat metric can be understood as the metric naturally preferred by the M5-brane low energy effective equations of motion and providing the propagation cone for all perturbative degrees of freedom). This remains an important problem for the future.

\section{Note added:}

During the completion of this paper the preprint 28 appeared, discussing an interesting $A d S_{3}$ self-dual string phase of OM-theory. In this preprint a conformal factor of the open membrane metric was presented that differed from ours. More recently this issue was resolved when the authors of [28] corrected their conformal factor, obtained using a different method, which now agrees with our result.

\section{Acknowledgments}

It is a pleasure to thank Jianxin Lu, Eric Bergshoeff, David Berman and especially Per Sundell for interesting discussions. The Groningen Institute for Theoretical Physics is acknowledged for their hospitality during which part of this work was done. 


\section{References}

[1] N. Seiberg and E. Witten, String theory and noncommutative geometry, JHEP 09 (1999) 032, hep-th/9908142.

[2] E. Bergshoeff, D. S. Berman, J. P. van der Schaar, and P. Sundell, A noncommutative m-theory five-brane, Nucl. Phys. B590 (2000) 173-197, hep-th/0005026].

[3] G. W. Gibbons and C. A. R. Herdeiro, Born-infeld theory and stringy causality, Phys. Rev. D63 (2001) 064006, hep-th/0008052.

[4] G. W. Gibbons and P. C. West, The metric and strong coupling limit of the m5-brane, hep-th/0011149.

[5] N. Seiberg, L. Susskind, and N. Toumbas, Space/time non-commutativity and causality, JHEP 06 (2000) 044, hep-th/0005015.

[6] J. L. F. Barbon and E. Rabinovici, Stringy fuzziness as the custodian of time-space noncommutativity, Phys. Lett. B486 (2000) 202-211, hep-th/0005073.

[7] R. Gopakumar, J. Maldacena, S. Minwalla, and A. Strominger, S-duality and noncommutative gauge theory, JHEP 06 (2000) 036, [hep-th/0005048].

[8] N. Seiberg, L. Susskind, and N. Toumbas, Strings in background electric field, space/time noncommutativity and a new noncritical string theory, JHEP 06 (2000) 021, hep-th/0005040].

[9] R. Gopakumar, S. Minwalla, N. Seiberg, and A. Strominger, Om theory in diverse dimensions, JHEP 08 (2000) 008, hep-th/0006062].

[10] E. Bergshoeff, D. S. Berman, J. P. van der Schaar, and P. Sundell, Critical fields on the m5-brane and noncommutative open strings, Phys. Lett. B492 (2000) 193-200, hep-th/0006112.

[11] A. A. Tseytlin, Born-infeld action, supersymmetry and string theory, hep-th/9908105.

[12] P. S. Howe, E. Sezgin, and P. C. West, Covariant field equations of the m-theory five-brane, Phys. Lett. B399 (1997) 49-59, hep-th/9702008].

[13] P. S. Howe, E. Sezgin, and P. C. West, The six-dimensional self-dual tensor, Phys. Lett. B400 (1997) 255-259, hep-th/9702111.

[14] G. W. Gibbons and D. A. Rasheed, Sl(2,r) invariance of non-linear electrodynamics coupled to an axion and a dilaton, Phys. Lett. B365 (1996) 46-50, hep-th/9509141.

[15] A. A. Tseytlin, Self-duality of born-infeld action and dirichlet 3-brane of type iib superstring theory, Nucl. Phys. B469 (1996) 51-67, hep-th/9602064. 
[16] D. Berman, Sl(2,z) duality of born-infeld theory from non-linear self-dual electrodynamics in 6 dimensions, Phys. Lett. B409 (1997) 153-159, hep-th/9706208.

[17] D. Berman, M5 on a torus and the three brane, Nucl. Phys. B533 (1998) 317-332, hep-th/9804115.

[18] M. Cederwall and A. Westerberg, World-volume fields, sl(2,z) and duality: The type iib 3- brane, JHEP 02 (1998) 004, hep-th/9710007].

[19] J. X. Lu, S. Roy, and H. Singh, Sl(2,z) duality and 4-dimensional noncommutative theories, Nucl. Phys. B595 (2001) 298-318, hep-th/0007168.

[20] J. G. Russo and M. M. Sheikh-Jabbari, On noncommutative open string theories, JHEP 07 (2000) 052, hep-th/0006202.

[21] T. Kawano and S. Terashima, S-duality from om-theory, Phys. Lett. B495 (2000) 207-214, hep-th/0006225.

[22] U. Gran and M. Nielsen, Non-commutative open (p,q)-string theories, hep-th/0104168.

[23] J. X. Lu, (1+p)-dimensional open d(p-2) brane theories, hep-th/0102056.

[24] H. Larsson and P. Sundell, Open string/open d-brane dualities: Old and new, hep-th/0103188.

[25] J. H. Schwarz, An sl(2,z) multiplet of type iib superstrings, Phys. Lett. B360 (1995) 13-18, hep-th/9508143.

[26] E. Bergshoeff and J. P. van der Schaar, Work in progress, .

[27] D. S. Berman et. al., Holographic noncommutativity, hep-th/0011282.

[28] D. S. Berman and P. Sundell, Ads(3) om theory and the self-dual string or membranes ending on the five-brane, hep-th/0105288. 